\title{
A review of optimal operation of microgrids
}

\author{
N. Karthik ${ }^{1}$, A. K. Parvathy ${ }^{2}$, R. Arul ${ }^{3}$ \\ ${ }^{1,2}$ Department of Electrical and Electronics Engineering, Hindustan Institute of Technology and Science, India \\ ${ }^{3}$ School of Electrical Engineering, Vellore Institute of Technology, India
}

\begin{tabular}{l}
\hline \hline Article Info \\
\hline Article history: \\
Received Jun 17, 2019 \\
Revised Dec 1, 2019 \\
Accepted Dec 10, 2019 \\
\hline
\end{tabular}

Keywords:

Distributed generation

Microgrid

Optimal generation scheduling

Optimization techniques

\begin{abstract}
The term microgrid refers to small-scale power grid that can operate autonomously or in concurrence with the area's main electrical grid. The intermittent characteristic of DGs which defies the power quality and voltage manifests the requirement for new planning and operation approaches for microgrids. Consequently, conventional optimization methods in new power systems have been critically biased all through the previous decade. One of the main technological and inexpensive tools in this regard is the optimal generation scheduling of microgrid. As a primary optimization tool in the planning and operation fields, optimal operation has an undeniable part in the power system. This paper reviews and evaluates the optimal operation approaches mostly related to microgrids. In this work, the foremost optimal generation scheduling approaches are compared in terms of their objective functions, techniques and constraints. To conclude, a few fundamental challenges occurring from the latest optimal generation scheduling techniques in microgrids are addressed.
\end{abstract}

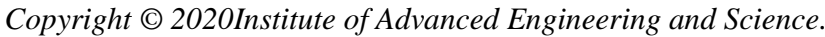
All rights reserved.

Corresponding Author:

N. Karthik,

Departement of Electrical and Electronics Engineering,

Hindustan Institute of Technology and Science,

No.1, Rajiv Gandhi Salai, Padur, Chennai, Tamilnadu, India.

Email: nkarthik@hindustanuniv.ac.in

\section{INTRODUCTION}

Owing to the speedy growth of utilization of Distributed Generations (DGs) in microgrids, their various aspects have been the concern of latest research. Microgrid is an dynamic distribution network which comprises together loads and Distributed Generations (DGs) and can operate in stand-alone mode or gridconnected mode [1]. The resources of distributed generation are non-conventional energy sources in order to curtail the use of fossil fuels. The distributed generation is incorporated into the main grid by means of intelligent micro-grid. With added distributed generation integrated into grid, it is significant to find out the best possible electrical power generation from each distributed generation in order that the electrical power needs can be convened with minimum emission and operational cost [2]. The optimal operation of microgrid is very important since it utilizes limited sources of energies. Several studies are carried out and reported in the area of optimal generation scheduling [3-6].

Optimal placement of DGs for loss minimization [7], reliability improvement [8] and energy cooperation optimization [9] are a few aspects of optimal scheduling in microgrids. Moreover, [10, 11] recommend different techniques for optimal operation of microgrids in both grid-connected and stand-alone modes. Small-scale wind turbines and solar photovoltaic panels generate DC power. Fuel cells, super capacitors and batteries store energy as DC. In addition, large quantity of energy distributed as AC is currently consumed as DC [12]. Opportunities subsist to exploit on the benefits of DC microgrids. DC microgrids are compatible to connect DC output types of distributed energy resources, and are suitable to protect sensitive loads from disturbances and power outages for instances voltage sags and swells [13]. Furthermore, DC microgrids comprise simpler power electronic interfaces and less points of failure [14]. 
In a DC microgrid, energy storage and a huge part of the sources and loads are interconnected by means of one or more DC buses. On the other hand, an AC grid is still required in view of the fact that some loads and sources cannot be directly connected to DC buses [15]. As a result, in the near future, DC microgrids are well thought-out as part of the main AC grid [16], where these two networks are linked to each other using the AC-DC converters to transmit power between them [17, 18]. When an AC grid is linked to one or more DC microgrids, the OPF problem of the AC-DC network acquires the structure of a non-convex optimization problem comprising DC microgrid and traditional AC power grid power flow equations, as well as the constraints entailed by the AC-DC power converters equations [19-21]. The non-convexity of the optimization problem occurs from the nonlinear power flow equations and quadratic dependence on the set of bus voltages. The problem may have multiple local optimal solutions [22]. Accordingly, a microgrid has high control capability and flexibility in terms of power quality and power system reliability [23-25]. In general, the operational modes of microgrids can be categorized as grid-connected or islanded mode. In the islanded mode, a microgrid should be stable whereas it is disconnected from the power grid. In addition, the function of DERs is significant [26].In the grid-connected mode, the main grid operates as a supporter which microgrid can send/receive electrical power to/from it. Central Controller (CC) and Microsource Controller (MC) [27-30] handle and manage the grids at various modes. Consequently, shifting from the grid connected mode to islanded mode can be carried out in two methods: full separation of the public grid and segregation of every individual feeder. In this structure, the most important function of $\mathrm{MC}$ is direct control of voltage level and power flow of connected loads to the grid at any circumstances. Direct control specifies that MC can be operated individually from CC if necessary. In addition, MC can take part in Economic load Dispatch (ELD) and Demand-Side Management (DSM) through controlling the renewable energy sources. In this situation, $\mathrm{CC}$ transmits control commands through MC [31, 32]. In this regards, one of the primary commands is the optimal scheduling of microgrid. In view of the fact that, one of the most important objectives along with the system operators is to reduce the microgrid cost, subsequently they should be able to think about and compare the energy cost of the major utility and the generation cost of the microgrid units despite the fact that satisfying all constraints in the grid-connected mode. In [33], a grid connected microgrid comprising battery storage system and photovoltaic system is developed to comply with the capmus load demand.

\section{MOTIVATION}

One of the most significant technological and cost-effective tools in power systems is optimal generation scheduling. By means of this part of software, control variables associated with the power system planning and operation, at a particular time, are found out in turn to accomplish a particular objective and ensure technical viability of the steady-state control actions. The optimal operation of microgrids is considered as a new advancement in power system studies [34, 35]. Therefore, an articulate categorization of these approaches required at this point of technology development. Furthermore, no widespread research has been so far conducted on microgrids.

\subsection{Scope}

This paper reviews and compares the different optimization techniques applied for achieving optimal operation of microgrids from diverse perceptions. At the same time as it is reported, the foremost approaches are compared in terms of Objective Functions (OFs), optimization techniques, constraints in addition to computational performances.

\section{OPTIMAL OPERATION OF MICROGRID}

Debapriya Das[36] formulated an economic load dispatch problem of a microgrid using four different optimization algorithms. The genrating cost of the dispatchable DGs present in the microgrid is taken as objective function. In [37], authors have inspected the effect of these constraints on two different test systems. Simulation results show that lambda logic technique has the fastest computational time.

S. Surender Reddy [38] proposed the optimal generation scheduling problem for a microgrid consisting of conventional generators, solar photovoltaic (PV) systems, wind turbine generators, electric vehicles (EV) and battery storage. Application results of the optimal generation scheduling of the microgrid with and without EVs and battery storage are attained for comparison. Simulation results reveals that the optimum cost incurred in microgrid with EVs and battery storage is less.

Reza Roofegari Nejad [39] proposed a new model for optimal operation of a microgrid comprising wind turbine, microturbine, energy storage system and loads. Particle Swarm Optimization (PSO) algorithm was used to optimize the operation of this microgrid. Alternatively, Monte Carlo simulation method has been 
applied, so as to model the uncertainties of wind generation, power consumption of uncontrollable loads, energy price of the upstream distribution network in addition to the disconnection probability from the network and failure probability of units. This method encompassed all probabilistic conditions and at last presented a probability distribution function for all the decision variables. Simulation results showed that using deterministic method in the optimal operation of microgrids with non-dispatchable resources was not appropriate and stochastic methods must be applied.

Pierluigi Siano [40] proposed multi objective and stochastic problem for optimal scheduling of microgrid comprising electrical and thermal loads, conventional energy sources (micro turbine and boiler), non- conventional energy sources (PV and wind), combined heat and power (CHP), energy storage systems (electrical and thermal storages) and series flexible alternating current transmission system (FACTS) devices. In order to attain a higher power transfer to the upstream grid Dynamic Voltage Restorer (DVR) is incorporated in the line between the main network and the microgrid. In the proposed optimization technique, solar radiation, wind speed and loads are considered as uncertain parameters based on a stochastic approach. The proposed stochastic and multi objective optimization problem is solved by using the augmented Epsilonconstraint technique. To reveal the efficacy and viability of the proposed optimization technique simulation results are compared with results attained using genetic algorithm.

Seyed Masoud Moghaddas Tafreshi [41] proposed a probabilistic Unit Commitment(UC) model for optimal operation of plug-in electric vehicles(PEVs) in microgrid. The microgrid considered here comprises of wind turbine, microturbines, PEVs, boiler, battery storage and thermal storage. The expected total profit of the UC schedule was taken as objective function. Particle Swarm Optimization (PSO) algorithm is applied to minimize the fitness function. Even though probabilistic UC-Vehicle to Grid (V2G) cannot absolutely represent the indeterminate nature of load, wind and vehicles, the attained values are nearer to reality in association with the deterministic ones. Comparing the simulation results of deterministic and probabilistic UC- V2G reveals that the probabilistic method does not overrate the total expected profit.

In [42], optimal management strategy of wind/PV/diesel independent hybrid systems for supplying required energy in autonomous microgrids is proposed. Guaranteed convergence Particle Swarm Optimization with Gaussian Mutation (GPSO-GM), is developed to solve the optimization problem. To demonstrate the effectiveness and validity of GPSO-GM results obtained are compared with results obtained by using Particle Swarm Optimization and Genetic Algorithm. Simulation results demonstrates that the design of hybrid energy systems based on using both battery banks and diesel generators to support non-conventional energy sources is more efficient than the design which only uses exclusively battery banks or diesel generators.

Optimal operation of microgrids considering the uncertainty of non-conventional energy genration was presented by Byung Ha Lee [43]. Simulation results reveal that stochastic methodology can be applied successfully for optimal operation of a microgrid with uncertainties through the case study. In [44], near optimal operation/allocation of Grid-level battery energy storage system (BESS) has been investigated with the deliberation of lifetime characteristics. Simulation results reveal that the ADP can optimize the system operation under various scenarios. In [45], Ketan P. Detroja have proposed a optimization-based MG framework for optimal operation of microgrid. The proposed optimization framework comprises of three optimization components to carry out unit commitment, consumer load scheduling and power balancing. The optimization problem is developed with the consideration of transmission constraints, ramp-up/rampdown constraints etc.,

Bo $\mathrm{Hu}$ [46] proposed an economic operation model of isolated community microgrid comprising micro-gas turbine, wind turbine, heat pump and energy storage battery. The optimization problem is solved using hybrid PSO technique. Simulation results reveal that temperature adjustment of temperature controlling devices can lessen charge-discharge cycles of the energy storage system and enhance microgrid schedulability besides improving the economic efficiency of the microgrid.

Peng Li [47] proposed improved bat algorithm along with point estimate method to optimize the operation of microgrid comprising wind turbine generator, solar photovoltaic system, micro turbine, fuel cell and battery. G. Liu [48] proposed an optimal scheduling strategy for microgrid operation considering constraints of islanding capability. A new concept called probability of successful islanding (PSI) is developed. The proposed chance-constrained model has two advantages when compared to the deterministic model. Tianguang Lv [49] proposed a multi-objective bi-level optimal operation model for distribution network with grid-connected microgrids to obtain operation benefits of both distribution network and microgrids. Simulation studied was carried out in IEEE 33-bus distribution network with Europe typical microgrid and a real system which has 128 nodes and 7 microgrids in Shandong, China.

In [50], a cost-effective hybrid power system in a coastal area of Bangladesh is proposed which minimizes gas emission by a substantial amount owing to reduced fuel consumption. HOMER software is used to determine the reduction in gas emission. Daniel Moga [51] formulated an optimization model based 
on the day-ahead forecasted power of noncontrollable loads at each time interval of the day (the load profile for the equipment of a greenhouse) in addition to the weather forecast based estimation of the solar energy availability. The optimization time period is one day ( 24 hours) that is divided into hourly slots. The optimization model aims to optimize the operation of three non-conventional energy sources (biogas, photovoltaic, geothermal) reducing the daily costs which are necessary for the dispatchable generators. In order to validate the results obtained, an experimental system equipped with smart metering instruments is introduced.Load control algorithm is implemented to accommodate PV generation operating in intentionalislanding mode [52]. LABVIEW software is used to design the load control algorithm.

Godfrey Gladson Moshi [53], presented a two Mixed-Integer Linear Programming (MILP) models for a complete microgrid planning under uncertainties in solar irradiance, electricity demand and wind speed. To illustrate and compare the effectiveness of the RO and 2SSIP model, the author presented a case study in which the two models are applied to plan a standalone microgrid in Singida, Tanzania. In [54], Nnamdi I. Nwulu proposed optimal economic dispatch of a grid connected microgrid. The microgrid comprises wind, solar photovoltaic and diesel power sources. Simulation results reveral that lower costs are attained in the microgrid when the grid operators DR benefit is maximized at the outlay of minimizing transaction/ fuel costs.

In [55], Sirus Mohammadi presented optimal operation management of microgrids using the point estimate method and firefly algorithm considering uncertainties in probabilistic energy management systems. Simulation results revealed that if the uncertain parameters considered can be calculated or projected, the distributions of all of the optimal costs and state variables can be precisely and proficiently evaluated by means of Hong's point estimate method. Jordan Radosavljevic [56] presented an efficient algorithm based on particle swarm optimization (PSO) for energy and operation management (EOM) of a microgrid comprising various distributed generation units and energy storage devices. PSO is applied to sort out the optimization problem. The obtained simulation results substantiated the efficiency of the proposed approach to sort out both probabilistic and deterministic EOM problems under various equipped scenarios of the microgrid.

Jingrui Zhang [57] proposed optimal day-ahead scheduling model for a microgrid system with wind turbine units, photovoltaic cells, battery storage systems and diesel generators. Simulation has been performed on three different IEEE standard bus systems. Simulation results reveal that for the optimal day-ahead scheduling of microgrids, the proposed optimization technique is consistent under both normal and fault operation conditions. Ninet Mohamed Ahmed [58] et.al., presented a comparative study between three dissimilar configurations for supplying an irrigating pumping system and a farmer's house with the required electrical demand in two different regions. HOGA (Hybrid Optimization by Genetic Algorithms) simulation software tool is utilized for optimal sizing and cost-effective analysis of hybrid standalone photovoltaic-wind system.

In [59], Yan Zhang presented a model predictive control (MPC) based optimal operation approach for residential microgrid with considering forecast uncertainties. The control accomplishment at each sampling time is attained by solving a novel mixed integer linear programming (MILP) optimization problem. Simulation results specify that the operation cost of MPC approach is appreciably lower than conventional day-ahead programming approach under perfect forecasting situation. In [60], Cuckoo Search Algorithm (CSA) has been implemented for solving the environmental economic dispatch problem of microgrid. Simulation result obtained from the CSA is compared with PSO and it signifies that the CSA method offers better solution when compared to PSO method.

In [61], Milana Trifkovic presented a parametric programming based approach for energy management in microgrids. The optimization problem is solved offline on a flexible time-scale basis, permitting online realization to be attainable on real-time system state updates. By making use of operational and design boundaries on the renewable energy systems, renewable resource inconsistency is captured as different parametric apprehensions of solar and wind power, which results in the conversion of the problem from nonlinear to a linear form. The algorithm was tested using various electricity pricing information to construct two case studies for incentivized and open market operations of the system. Both case studies are applied to the same renewable energy apprehensions to optimize the decisions of a microgrid over a one week operational period. Simulation results reveal that under the incentivized program, the storage system is almost not utilized and most power production extras to local demand is sold to the main grid.

A DC microgrid with improved Maximum Power Point Tracking (MPPT) algorithms for solar and wind energy systems is developed in [62]. A two-model MPPT technique is implemented to improve the PV system power generation. In addition, an Optimal Power Control MPPT algorithm is included for the Wind Energy Conversion System (WECS) with pitch angle controlling method. To improve the supply to the grid Space Vector Pulse Width Modulation technique is implemented. In [63], Ango Sobu presented an optimal operation planning for an isolated microgrid which comprises photovoltaic power generators, wind turbine, diesel generators and batteries. This optimization problem is solved using Particle Swarm Optimization 
(PSO). Simulation results reveal that even though the operation cost of the operation planning attained with indeterminate cost model is greater than that with indiscriminate cost model.

In [64], microgrid stochastic economic load dispatch (SELD) problem is devised based on the waitand-see approach. Simulation results reveal that the new mechanism in IPSO adds to the optimization capability. Sajid Hussain Qazi1 et. al., [65] developed a PI controller based voltage controller to improve voltage profile of islanded microgrid. In [66], a power allocation approach for storage batteries and diesel generators is proposed by means of the overall deliberation of the financial and ecological benefits of system operation. The optimization problem is solved by the non-dominated sorting genetic algorithm (NSGA-II). The model is analyzed by solving a problem on a realistic island, and the sagacity of the proposed model and the power allocation approach is confirmed. In [67], Abdorreza Rabiee presented the instantaneous scheduling of electrical vehicles and receptive loads to minimize operation cost and emission in occurrence of PV and wind powers in microgrid. Simulation results revealed that the integration of electrical vehicles and reactive loads shows the way to diminish the system emission and operation costs. In [68], Interior Search Algorithm was applied to elucidate the economic load dispatch problem in a microgrid.

\section{CONCLUSION}

From the time when the publication of the first optimal generation scheduling method for bulk power systems, several contributions to the improvement of basic idea of optimal operation have been proposed to suit the requirements of several applications. The arrival of microgrids and then smart grids with their distinctive features and infrastructures to overcome most of the equipped analysis for instance optimal generation scheduling has added a new chapter to the field of power systems. The superior performance of optimal generation scheduling approaches in microgrids has paid attention to researchers and power system companies all over the world. In this field, various optimization techniques, objective functions, and constraints are recommended. In reality, this research reviewed and compared optimal generation scheduling approaches of microgrids from various perspectives with the intention of providing an overall vision of this optimization problem. This classification and analysis assist researchers to figure out all of them. Generally, regardless of the optimization algorithm used to elucidate the optimal generation scheduling problem, the models until now developed have as a minimum one of the subsequent specifications. Most of the reported approaches, have considered the microgrid in a grid-connected mode. Furthermore, all optimization techniques have considered the power generation limits in the set of constraints. In addition, these studies examined the appropriate synchronization among conventional control variables with DG controls. The review illustrates that most of the approaches have considered the microgrids as unbalanced distribution systems. So, a more effective optimization method should be employed. As a result, each power system type has a set of optimization approaches which is more suitable for its purpose. Challenges in the optimal operation of microgrid: New appropriate and comprehensive analysis software; Novel and widespread meta-heuristic optimization techniques to solve optimal generation scheduling problems; Modeling of uncertainties in the generation of renewable energy sources; Utilization of new components for instance storage systems; Optimal operation of microgrid in an unbalanced system.

\section{REFERENCES}

[1] Ackermann T., Andersson G., Soder L., "Distributed generation: a definition," Electric Power Systems Research, vol. 57, no. 3, pp. 195-204, April 2001.

[2] Bouzid A. M., Guerrero J. M., Cheriti A., Bouhamida M, Sicard P, Benghanem M.,“A survey on control of electric power distributed generation systems for microgrid applications," Renewableand Sustainable Energy Reviews, vol. 44, pp. 751-66, April 2015.

[3] N. W. A. Lidula and A. D. Rajapakse, "Microgrids research: A review of experimental microgrids and test systems," Renewable and Sustainable Energy Reviews, vol. 15, no. 1, pp. 186-202, January 2011.

[4] A. K. Basu, S. P. Chowdhury, S. Chowdhury, and S.Paul, Microgrids: Energy management by strategic deployment of DERs-A comprehensive survey,"Renewable and Sustainable Energy Reviews," vol. 15, no. 9, pp. 4348-4356, December 2011.

[5] W. Gu, Z. Wu, R. Bo, W. Liu, G. Zhou, W. Chen, et al., "Modeling, planning and optimal energy management of combined cooling, heating and power microgrid: A review," International Journal of Electrical Power \& Energy Systems, vol. 54, pp. 26-37, January 2014.

[6] S. Mohammadi, S. Soleymani, and B. Mozafari, "Scenario-based stochastic operation management of MicroGrid including Wind, Photovoltaic, Micro-Turbine, Fuel Cell and Energy Storage Devices," International Journal of Electrical Power \& Energy Systems, vol. 54, pp. 525-535, January 2014.

[7] P. Vijay Babu and S.P. Singh, "Optimal Placement of DG in Distribution network for Power loss minimization using NLP \& PLS Technique,” Energy Procedia, vol. 90, pp. 441-454, December 2016. 
[8] Seyed Ali Arefifar, Yasser Abdel-Rady I. Mohamed, "DG Mix, Reactive Sources and Energy Storage Units for Optimizing Microgrid Reliability and Supply Security," in IEEE Transactions on Smart Grid, vol. 5, no. 4, pp. 1835-1844, July 2014.

[9] Katayoun Rahbar, Member, IEEE, Chin Choy Chai, Member, IEEE, and Rui Zhang, "Energy Cooperation Optimization in Microgrids with Renewable Energy Integration," in IEEE Transactions on Smart Grid, vol. 9, no. 2, pp. 1482-1493, March 2018.

[10] Quanyuan Jiang, MeidongXue, GuangchaoGeng, "Energy Management of Microgrid in Grid-Connected and StandAlone Modes," in IEEE Transactions on Power Systems, vol. 28, no. 3, pp. 3380-3389, Aug. 2013.

[11] M. Mohammad, "Optimal Operation Management of a Typical Microgrid as Grid Connected in Power Systems Using Fuzzy Sliding-Mode Control (FSMC) Approach," World Applied Sciences Journal, vol. 28, no. 4, pp. 440-448, 2013.

[12] G. T. Heydt, "The Next Generation of Power Distribution Systems," in IEEE Transactions on Smart Grid, vol. 1, no. 3, pp. 225-235, Dec. 2010.

[13] Ahmed T. Elsayed, Ahmed A. Mohamed, Osama A. Mohammed, "DC microgrids and distribution systems: An overview," Electric Power Systems Research, vol. 119, pp. 407-417, Febuary2015.

[14] C.N. Papadimitriou, E.I. Zountouridou, N.D. Hatziargyriou, "Review of hierarchical control in DC microgrids," Electric Power Systems Research, vol. 122, pp. 159-167, May 2015.

[15] Jackson John Justo, Francis Mwasilu, JuLee, Jin-Woo Jung, “AC-microgrids versus DC-microgrids with distributed energy resources: A review," Renewable and Sustainable Energy Reviews, vol. 24, no. C, pp. 387-405, 2013.

[16] EstefaníaPlanas, Jon Andreu, Jose IgnacioGarate, IñigoMartínez de Alegría, Edorta Ibarra, "AC and DC technology in microgrids: A review," Renewable and Sustainable Energy Rev., vol. 43, pp. 726-749, March 2015.

[17] M. Shahbazi, A. Khorsandi, "Power electronic converters in microgrid applications," Microgrid, vol. 10, pp. 281-309, 2017.

[18] S.Khosrogorji, M. Ahmadian, H. Torkaman, S. Soori, "Multi-input DC/DC converters in connection with distributed generation units - A review," Renewable and Sustainable Energy Reviews, vol. 66, pp. 360-379, December 2016.

[19] T. Dragicevic, F. Blaabjerg, "Power Electronics for Microgrids: Concepts and Future Trends," Microgrid, pp. 263-279, 2017.

[20] C. Chakraborty, H. H. Iu and D. Dah-Chuan Lu, "Power converters, control, and energy management for distributed generation," in IEEE Transactions on Industrial Electronics, vol. 62, no. 7, pp. 4466-4470, July 2015.

[21] Y. Liao, "A Novel Reduced Switching Loss Bidirectional AC/DC Converter PWM Strategy With Feedforward Control for Grid-Tied Microgrid Systems," in IEEE Transactions on Power Electronics, vol. 29, no. 3, pp. 1500-1513, March 2014

[22] VahidHosseinnezhad, Mansour Rafiee, Mohammad Ahmadian, PierluigiSiano, "Optimal day-ahead operational planning of microgrids," Energy Conversion and Management, vol. 126, pp. 142-157, October 2016.

[23] AbdollahKavousi-Fard, Amin Khodaei, Shay Bahramirad, "Improved efficiency, enhanced reliability and reduced cost: The transition from static microgrids to reconfigurable microgrids," The Electricity Journal, vol. 29 no. 10, pp. 22-27, December 2016.

[24] Mohamad Sadeghian, BahadorFani, "Advanced localized reactive power sharing in microgrids," Electric Power System Research, vol. 151, pp. 136-148, October 2017.

[25] Ahmed Alabdulwahab, Mohammad Shahidehpour, "Microgrid networking for the monitoring, control and protection of modern power systems," The Electricity Journal, vol. 29, no. 10, pp. 1-7, December 2016

[26] NurNajihah Abu Bakar, Mohammad Yusri Hassan, Mohamad FaniSulaima, Mohamad Na'im Mohd Nasir, AziahKhamis, "Microgrid and load shedding scheme during islanded mode: A review," Renewable and Sustainable Energy Reviews, vol. 71(C), pp. 161-169, 2017.

[27] Karim Hassan Youssef, "Optimal management of unbalanced smart microgrids for scheduled and unscheduled multiple transitions between grid-connected and islanded modes," Electric Power Systems Research, vol. 141, pp. 104-113, December 2016.

[28] Majid Mehrasa, EdrisPouresmaeil, Bo Norregaard Jorgensen, Joao, P.S.Catalao, "A control plan for the stable operation of microgrids during grid-connected and islanded modes," Electric Power Systems Research, vol. 129, pp. 10-22, 2015.

[29] Hossam A.Gabbar, Abdelazeem A.Abdelsalam, "Microgrid energy management in grid-connected and islanding modes based on SVC," Energy Conversion and Management, vol. 86, pp. 964-972, October 2014.

[30] Omid Palizban, Kimmo Kauhaniemi, "Hierarchical control structure in microgrids with distributed generation: Island and grid-connected mode," Renewable and Sustainable Energy Reviews, vol. 44, pp. 797-813, April 2015.

[31] Amandeep Kaur, Jitender Kaushal, Prasenjit Basak, "A review on microgrid central controller," Renewable and Sustainable Energy Reviews, vol. 55, pp. 338-345, March 2016.

[32] M.S. Mahmoud, "Microgrid Control Problems and Related Issues," Microgrid, vol. 1, pp. 1-42, 2017.

[33] Mohammed ReyasudinBasir Khan, JagadeeshPasupuleti, Jabbar Al-Fattah, MehrdadTahmasebi, "Optimal GridConnected PV System for a Campus Microgrid," Indonesian Journal of Electrical Engineering and Computer Science, vol. 12, no. 3, pp. 899-906, December 2018.

[34] B. Khorramdel, H. Khorramdel, H. Marzooghi, "Multi-Objective Optimal Operation of Microgrid with an Efficient Stochastic Algorithm Considering Uncertainty of Wind Power," in International Review on Modelling and Simulations, vol. 4, no. 6, pp. 3079-3089, December 2011.

[35] AvirupMaulik, Debapriya Das, "Optimal operation of microgrid using four different optimization techniques," Sustainable Energy Technologies and Assessments, vol. 21, pp. 100-120, June 2017. 
[36] Samir M. Dawoud, Lin Xiangning, Firas M. F. Flaih, Merfat I. Okba, "PSO Algorithm for Optimal Placement of Multiple SPV Based Distributed Generators in Microgrids," 2016 IEEE PES Asia-Pacific Power and Energy Engineering Conference (APPEEC), Xi'an, pp. 125-129, 2016.

[37] NimaNikmehr, SajadNajafiRavadanegh, "Heuristic probabilistic power flow algorithm for microgrids operation and planning," in IET Generation, Transmission \& Distribution, vol. 9, no. 11, pp. 985-995, 2015.

[38] S. Surender Reddy, Jae Young, Chan Mook Jung, "Optimal operation of microgrid using hybrid differential evolution and harmony search algorithm," Springer Frontiers in Energy, vol. 10, no. 3, pp. 355-362, 2016.

[39] R. RoofegariNejad, S. M. Hakimi, S. M. MoghaddasTafreshi, "A Novel Demand Response Method for Smart Microgrids Related to the Uncertainties of Renewable Energy Resources and Energy Price," Journal of Electrical Systems, vol. 12, no. 2, pp. 419-441, June 2016.

[40] Mehdi Ahmadi Jirdehi, VahidSohrabiTabar, Reza Hemmati, PierluigiSiano, "Multi objective stochastic microgrid scheduling incorporating dynamic voltage restorer," International Journal of Electrical Power \& Energy Systems, vol. 93, pp. 316-327, December 2017.

[41] Seyed Masoud Moghaddas Tafreshi, Hassan Ranjbarzadeh, Mehdi Jafari, Hamid Khayyam, "A probabilistic unit commitment model for optimal operation of plug-in electric vehicles in microgrid," Renewable and Sustainable Energy Reviews, vol. 66, pp. 934-947, December 2016.

[42] Mohammad Abedini, Mohammad H. Moradi, S. Mahdi Hosseinian Perninge, "Optimal management of microgrids including renewable energy scources using GPSO-GM algorithm,” Renewable Energy, vol. 90, pp. 430-439, May 2016.

[43] Byung Ha Lee, Jin Ah Yang, "A Study on Optimal Operation of Microgrids Considering the Uncertainty of Renewable Generation and Load by Use of Duration Curves," 2015 IEEE Power \& Energy Society General Meeting, Denver, CO, pp. 1-5, 2015

[44] Avijit Das, Zhen Ni, and XiangnanZhong, "Near Optimal Control for Microgrid Energy Systems Considering Battery Lifetime Characteristics,"2016 IEEE Symposium Series on Computational Intelligence (SSCI), Athens, pp. 1-8, 2016.

[45] Ketan P. Detroja, "Optimal autonomous microgrid operation: A holistic view,"Applied Energy, vol. 173, pp. 320-330, July 2016.

[46] Bo Hu, He Wang, Sen Yao, "Optimal economic operation of isolated community microgrid incorporating temperature controlling devices,"Protection and Control of Modern Power Systems, pp. 1-11, December 2017.

[47] Peng Li, Zeyuan Zhou, Ruyu Shi, "Probabilistic optimal operation management of microgrid using point estimate method and improved bat algorithm," 2014 IEEE PES General Meeting | Conference \& Exposition, National Harbor, MD, pp. 1-5, 2014.

[48] G. Liu, M. Starke, B. Xiao, X. Zhang, K. Tomsovic, "Microgrid Optimal Scheduling With Chance-Constrained Islanding Capability," Electric Power Systems Research, vol. 145, pp. 197-206, April 2017.

[49] TianguangLv, Qian Ai, Yuanyuan Zhao, "A bi-level multi-objective optimal operation of grid-connected microgrids,” Electric Power Systems Research, vol. 131, pp. 60-70, February 2016.

[50] Ahmed Zubair, Aman Abdulla Tanvir, Md. Mehedi Hasan, "Optimal Planning of Standalone Solar-Wind-Diesel Hybrid Energy System for a Coastal Area of Bangladesh," in International Journal of Electrical and Computer Engineering, vol. 2, no. 6, pp. 731-738, December 2012.

[51] Daniel Moga, DorinPetreuș, Vlad Mureșan, NicoletaStroia, Gloria Cosovici, "Optimal generation scheduling in islanded microgrids,” IFAC-PapersOnLine, vol. 49, no. 27, pp. 135-139, 2016.

[52] Mohamad Haireen Bin Fatheli, NurlzzatiZolkifri, Chin Kim Gan, Musa Bin Yusup Lada, "Development of Load Control Algorithm for PV Microgrid," International Journal of Electrical and Computer Engineering, vol. 7, no. 2, pp. 619-630, April 2017.

[53] Godfrey Gladson Moshi, Cristian Bovo, Alberto Berizzi, and Leonardo Taccari, "Optimization of integrated design and operation of microgrids under uncertainty," 2016 Power Systems Computation Conference (PSCC), Genoa, pp. 1-7. 2016.

[54] Nnamdi I. Nwulu, Xiaohua Xia, "Optimal dispatch for a microgrid incorporating renewables and demand response," J. Renewable Energy, vol. 101, pp. 16-28, Febuary 2017.

[55] Sirus Mohammadi, Babak Mozafari, Soodabeh Soleymani, "Optimal operation management of microgrids using the point estimate method and firefly algorithm whileconsidering uncertainty," in Turkish Journal of Electrical Engineering and Computer Sciences, vol. 22, no. 3, pp. 735-753, January 2014.

[56] Jordan Radosavljevic, Miroljub Jevtic, Dardan Klimenta, "Energy and operation management of a microgrid using particle swarm optimization. Engineering Optimization," in Engineering Optimization, pp. 1-20, June 2015.

[57] Jingrui Zhang, Yihong Wua, Yiran Guo, Bo Wang, Hengyue Wang, Houde Liu, "A hybrid harmony search algorithm with differential evolution for day-ahead scheduling problem of a microgrid with consideration of power flow constraints," Applied Energy, vol. 183, pp. 791-804, December 2016.

[58] Ninet Mohamed Ahmed, Hanaa Mohamed Farghally, Faten Hosney Fahmy, "Optimal Sizing and Economical Analysis of PV-Wind Hybrid Power System for Water Irrigation using Genetic Algorithm,” International Journal of Electrical and Computer Engineering (IJECE), vol. 7, no. 4, pp. 1797-1814, August 2017.

[59] Yan Zhang, Tao Zhang, Rui Wang, Yajie Liu, Bo Guo, "Optimal operation of a smart residential microgrid based on model predictive control by considering uncertainties and storage impacts," Solar Energy, vol. 122, pp. 1052-1065, December 2015.

[60] S. Vasanthakumar, N. Kumarappan, R. Arulraj and T. Vigneysh, R., "Cuckoo Search Algorithm based Environmental Economic Dispatch of Microgrid System with Distributed Generation," 2015 International 
Conference on Smart Technologies and Management for Computing, Communication, Controls, Energy and Materials (ICSTM), Chennai, pp. 575-580, 2015.

[61] Evar Chinedu Umeozor, Milana Trifkovic, "Operational scheduling of microgrids via parametric programming," Applied Energy, vol. 180, pp. 672-681, October 2016.

[62] D. ChinnaKullay Reddy, S. Satyanarayana, V. Ganesh, "Design of Hybrid Solar Wind Energy System in a Microgrid with MPPT Techniques," International Journal of Electrical and Computer Engineering, vol. 8, no. 2, pp. 730-740, April 2018.

[63] Ango Sobu, Guohong Wu, "Optimal operation planning method for isolated microgrid considering uncertainties ofrenewable power generations and load demand," IEEE PES Innovative Smart Grid Technologies, Tianjin, pp. 1-6, 2012.

[64] Yi Tan, Yijia Cao, Canbing Li, Yong Li, Li Yu, Zhikun Zhang and Shengwei Tang, "Microgrid stochastic economic load dispatch based on two-point estimate method and improved particle swarm optimization," International Transactions on Electrical Energy Systems, vol. 25, no. 10, June 2014.

[65] Sajid Hussain Qazi, M. W. Mustafa, "Improving Voltage Profile of Islanded Microgrid using PI Controller," International Journal of Electrical and Computer Engineering (IJECE), vol. 8, no. 3, pp. 1383-1388, June 2018.

[66] Qingfeng Tang, Nian Liu, and Jianhua Zhang, "Optimal Operation Method for Microgrid with Wind/PV/Diesel Generator/Battery and Desalination," Journal of Applied Mathematics, pp. 1-12, June 2014.

[67] Abdorreza Rabiee, Mohammad Sadeghi, Jamshid Aghaeic, Alireza Heidari, "Optimal operation of microgrids through simultaneous scheduling of electrical vehicles and responsive loads considering wind and PV units uncertainties," Renewableand Sustainable Energy Reviews, vol. 57, pp. 721-739, May 2016.

[68] N. Karthik, A. K. Parvathy, R. Arul, R, Jayapragash, Sathiya Narayanan, "Economic Load Dispatch in a Microgrid using Interior Search Algorithm," International Conference on Innovations in Power and Advanced Computing Technologies, i-PACT-2019, 2019. 